\title{
Acknowledgement to Reviewers of Machines in 2019
}

\author{
Machines Editorial Office \\ MDPI, St. Alban-Anlage 66, 4052 Basel, Switzerland \\ Published: 19 January 2020
}

The editorial team greatly appreciates the reviewers who have dedicated their considerable time and expertise to the journal's rigorous editorial process over the past 12 months, regardless of whether the papers are finally published or not. In 2019, a total of 76 papers were published in the journal, with a median time to first decision of 21 days and a median time from submission to publication of 50 days. The editors would like to express their sincere gratitude to the following reviewers for their generous contribution in 2019:

\author{
Abdullah, Nur Azam \\ Abu-Mahfouz, Issam \\ Adamus, Janina \\ Aliheidari, Nahal \\ Bai, Xianxu \\ Balandin, Dmitry V. \\ Barbon Jr., Sylvio \\ Bareille, Olivier A. \\ Barriot, Jean-Pierre \\ Bechtsis, Dimitrios \\ Belhocine, Ali \\ Berbyuk, Viktor \\ Berzi, Lorenzo \\ Bhaskaran, Gopalakrishnan \\ Bianchini, Alessandro \\ Bica, Ioan \\ Bin, Lin \\ Botta, Fabio \\ Brasil Cavalcante, André Luís \\ Cacciola, Stefano \\ Cammarata, Alessandro \\ Campatelli, Gianni \\ Canders, Wolf-Rüdiger \\ Cao, Hongrui \\ Cao, Wen-Ping \\ Cappello, Leonardo \\ Cardoso, José Roberto \\ Castelli-Dezza, Francesco \\ Chasalevris, Athanasios \\ Chávez, Esteban \\ Chen, Kuen-Suan \\ Chen, Tsung-Chia \\ Chen, Wujun
}

\author{
Choi, Seung-bok \\ Christophe, Sauvey \\ Chung, Tein-Yaw David \\ Cojocaru, Vasile \\ Cordeiro, Rafael \\ Corgnale, Claudio \\ Cruz, Pedro \\ Cui, Hao \\ De Falco, Domenico \\ Dehghanghadikolaei, Amir \\ Ding, Shihong \\ Ding, Songlin \\ Ding, Wenfeng \\ Dmitrievskii, Vladimir \\ Dobre, Alexandru \\ Dobreva, Antoaneta \\ Dragoi, Elena Niculina \\ Du, Yi \\ Duarte Marafona, José \\ Duguleană, Mihai \\ Dworak, Paweł \\ Eason, Paul D. \\ Esteban, M. Dolores \\ Evangelista, Stefania \\ Fanghella, Pietro \\ Favi, Claudio \\ Fedák, Viliam \\ Feliu, Vicente \\ Fitzgerald, Breiffni \\ Foszcz, Dariusz \\ Francalanza, Emmanuel \\ Frizziero, Leonardo \\ Furferi, Rocco
}


Galdos, Lander

Gallardo-Alvarado, Jaime

Galstyan, Vardan

Gamez-Montero, P.J.

Garrido Jurado, Juan

Gasiyarov, Vadim R.

Gazizulina, Albina

Gianluca, Alaimo

Giorgetti, Alessandro

Grossi, Niccolò

Guilbert, Damien

Guo, Jiang

Gusev, Michail

Hamdi, Hédi

Haupt, Michael

Hernando-Garcia, Jorge

Herrmann, Thomas

Hoyos Velasco, Fredy

Hsia, Shao-Yi

Hsiao, Chun-Ching

$\mathrm{Hu}$, Chuan

$\mathrm{Hu}$, Guobiao

Huang, Haihong

Huchet, Florian

Hueso, Miguel

Huston, Dryver

Huzlik, Rostislav

Idehara, Sérgio Junichi

Jackson, Mark

Jagiela, Mariusz

Jalil, Bushra

Jeong, Useok

Johnson, Mark E

Joles, Jaap A.

Jonak, Józef

Judek, Slawomir

Jurca, Florin

Kanarachos, Stratis

Kasprzyk, Jerzy

Kato, Hideaki

Kazak, Jan

Kefalas, Themistoklis

Kikuchi, Takehito

Kim, Jang Mok

Kitahara, Tatsumi

Koczara, Wlodzimierz

Kolasiński, Piotr

Królczyk, Grzegorz

Kunetsov, Vladimir

Lacerda, Márcio Júnior

Lara Molina, Fabian Andres

Lara, David
Latypov, Marat

Le, NamTuan

Lenzo, Basilio

Leonardi, Stefano

Levi, Alessandro

Li, Zhixiong

Liang, Zhiqiang

Lin, Yu-Chen

Linß, Sebastian

Lisovenko, Dmitry

Liu, Shengheng

Liu, Wei

López-Huerta, Francisco

Makowski, Michał

Małecki, Krzysztof

Markopoulos, Angelos

Martis, Claudia

Maurotto, Agostino

Mayr, Josef

Megahed, Mustafa

Miklosevic, Kresimir

Mikolajczyk, Tadeusz

Milic, Vladimir

Monkova, Katarina

Morańda, Hubert

Mureddu, Mario

Nassehi, Aydin

Nee, Andrew

Netto, Roberto S.

Nikolakopoulos, Pantelis G.

Nouri, Nima

Nuchkrua, Thanana

Orekhov, Andrew

Ouakad, Hassen

Padmanaban, Sanjeevikumar

Paleu, Viorel

Pan, Yunpeng

Pandkar, Anup

Papanikolaou, Nick

Pappalardo, Carmine Maria

Paredes, Manuel

Park, You-Jin

Parra, Vicente

Parrondo-Gayo, Jorge Luis

Perduková, Daniela

Pervaiz, Salman

Petrescu, Florian Ion Tiberiu

Piltan, Farzin

Portillo-Vélez, Rogelio De J.

Prakht, Vladimir

Precup, Radu-Emil

Rabinovici, Raul 
Radkowski, Rafael

Radulescu, Mircea

Rafael, Silviano

Rao, Dantam

Raush, Gustavo

Ravasio, Chiara

Reimer, Uwe

Ren, Yi

Richiedei, Dario

Rizzo, Rocco

Roger, Daniel

Rojas Sola, José

Romanowicz, Paweł

Rosati Papini, Gastone Pietro

Rosso, Carlo

Rubiec, Arkadiusz

Ruiz, Oscar

Salah, Wael

Sánchez, René-Vinicio

Savio, Gianpaolo

Schönemann, Lars

Semail, Eric

Shemelya, Corey

Shepherd, Jonathan

Siami, Ali

Simionescu, Petru

Socas, Rafael

Sohn, Jung Woo

Solitro, Giovanni Francesco

Somov, Yevgeny

Song, Ziyou

Stavropoulos, Panagiotis

Strazdienè, Eugenija

Świercz, Rafał

Szabo, Lorand

Sziebig, Gabor

Takeda, Yukio

Taler, Jan

Tappa, Karthik

Tavakoli, Ahmad

Taylor, Ambrose
Tekes, Ayse

Theodossiou, Nicolaos

Tiberiu Petrescu, Florian Ion

Tlelo-Cuautle, Esteban

Toboła, Daniel

Tomasz, Rymarczyk

Tomczak, Janusz

Torres, David

Trapp, Johannes

Tsao, Chung-Chen

Ullah, AMM Sharif

Valencia-Palomo, Guillermo

Valtierra-Rodriguez, Martin

Varma, Vijaykumar B.

Vázquez, Andrés S.

Velamati, Ratna Kishore

Verrelst, Björn

Vidakis, Nectarios

Višniakov, Nikolaj

Viveiros, Carla

Vlachos, Dimitrios

Wang, Kesheng

Wang, Kundong

Wang, Shuangyi

Wei, Wu

Wieringa, Fokko

Wiessner, Sven

Willert, Andreas

Wojciechowski, Szymon

Xiao, Shaoping

Xiong, Yangshou

Yunusa-Kaltungo, Akilu

Żak, Krzysztof

Zhang, Aron

Zhang, He

Zhang, Hong

Zhou, Jiehan

Zhou, Kan

Zhuang, Yan

Zolotová, Iveta

(C) 2020 by the author. Licensee MDPI, Basel, Switzerland. This article is an open access article distributed under the terms and conditions of the Creative Commons Attribution (CC BY) license (http://creativecommons.org/licenses/by/4.0/). 